\title{
Complicated acute mastoiditis with temporal subdural abscess and otogenic lateral sinus thrombosis: A case report
}

\author{
Enrico Maria Amadei* and Claudio Cola \\ Department of Otorhinolaryngology and Audiology, Infermi Hospital, Rimini, Italy
}

\begin{abstract}
Otitis is a very common disease in the pediatric population.Sometimes it is possible to have a serious complication such as otomastoiditis. This rarely can further complicate, leading to potentially fatal diseases, such as intracranial abscess and ipsilateral sigmoid sinus thrombosis. We describe the paradigmatic case of an 8 -yearold girl with complicated left mastoiditis. We report how we usually treat these young patients, emphasizing our diagnostic and therapeutic strategy. We conclude with the management doubts that are still under international discussion.
\end{abstract}

\section{Case report}

We describe a case of a 8-year-old female with an acute left otomastoiditis, accompanied by a left temporal extracerebral and subdural abscess with left paracebellar extension. The first manifestation was a left otitis, complicating an infection of the upper airways. Family doctor used cefixima, one administration per day. The Patient arrived at the hospital after 2 days, because of worsening of the symptoms with persistent otalgia, headache, vertigo and tendency to the asleep.

In consideration of the clinical picture, blood tests are performed. Antibiotic therapy is started with ceftriaxone and the patient is admitted for the necessary checks and treatments. Blood chemistry tests documented leukocytosis with lymphomonocytosis, with increased transaminases and increase in PCR. Due to the appearance of laboratory signs of cholestasis, we changed antibiotic therapy from ceftriaxone to ceftazidime. In the suspicion of mononucleosis, serology is performed for EBV with subsequent detection of positive IgM (negative IgG).

On the second day of hospitalization, the Patient got worse, with more tendency to fall asleep associated with mild pain in palpation of the left mastoid. We arrived at the diagnosis by performing a CT scan, finding a left coalescent mastoiditis, accompanied by a left temporal extracerebral and subdural abscess with left paracebellar extension (Figure 1). Then the Patient was subjected to an angio magnetic resonance, finding an intracranial thrombosis with failure to visualize the left sigmoid sinus and a flow reduction on the lateral sinus (Figure $2,3)$. So we performed the evacuation of the abscess by a retrosigmoid approach, and a left mastoidectomy by a retroauricolar approach. We made up a closed tympanoplasty, saving the stapes (Figure 4). We found a positive culture test for streptococcus agalactiae. Culture examination on ear pads was negative.

We used as antibiotic therapy metronidazole and vancomycin, while ceftazidime therapy was replaced with meropenem for the best penetration of the blood-brain barrier. After 10 days of therapy with meropenem it was attended appearance of a pruritic maculoeritematous exanthema spread to the whole body, compatible with crossreaction from EBV infection in progress. We treated this complication with a steroid therapy for 8 days. After 18 days of this therapy, the infectiousdisease doctor recommended the suspension of the triple therapy and

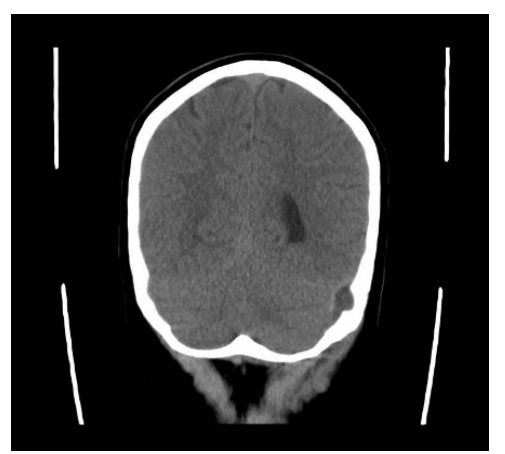

Figure 1. CT before surgery. Left temporal extracerebral and subdural abscess

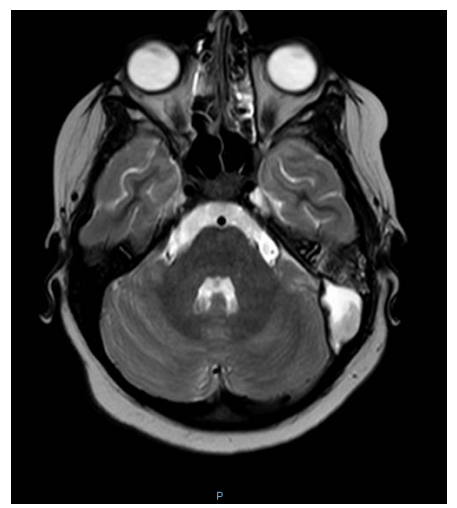

Figure 2. MRI before surgery. Left coalescent mastoiditis, accompanied by a left temporal extracerebral and subdural abscess with left paracebellar extension

*Correspondence to: Enrico Maria Amadei, Department of Otorhinolaryngology and Audiology, Infermi Hospital, via Popilia 233, Rimini, RN, 47922, Italy, Tel: +39 3283625727; E-mail: enricomaria.amadei@libero.it

Key words: complicated mastoiditis, temporal abscess, otogenic lateral sinus thrombosis, acute otitis media

Received: October 01, 2018; Accepted: October 16, 2018; Published: October 19,2018 
the use of clindamycin alone up to 4 weeks of intravenous antibiotic. Then we administered amoxicillin + clavulanic acid by mouth 3 times a day for another 3 weeks.

Regarding the anticoagulant therapy, we administered to the Pz low molecular weight heparin 4000 IU 2 times a day for 2 months, starting from the date of diagnosis of endocranial thrombosis. We found a recovery of cerebral vascular patency at magnetic resonance control carried out 1 month after drainage and mastoidectomy (Figure 5).

18 months later we executed the second programmed time via endoauricolar approach, in order to restore the auditory function. For ossiculoplasty we posizioned a titanium prosthesis (PORP: partial ossicular replacement prosthesis) between the stapes head and a tragal cartilage disc underlay the tympanic membrane. So we reached a good earing result with $\mathrm{ABG}$ (air bone gap) closed to $10 \mathrm{~dB}$.

\section{Discussion}

We know from Literature that mastoiditis complicates a middle otitis with a frequency of 1.2 to 3.8 cases per 100,000 children-year. It is

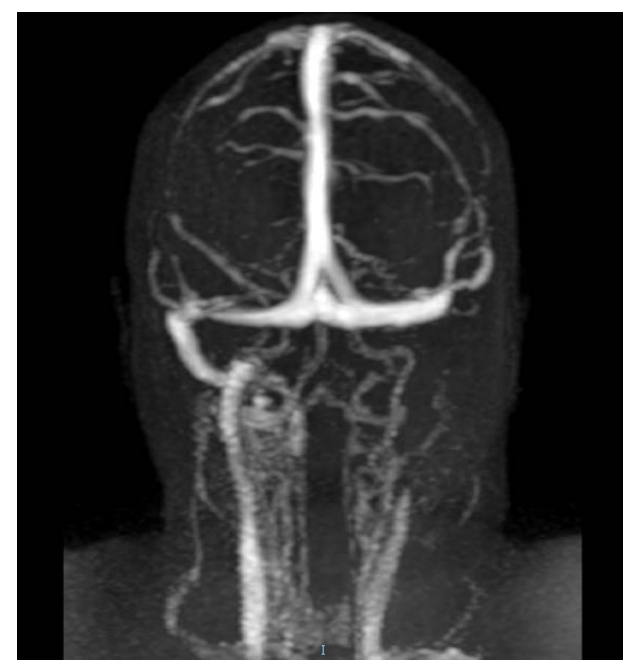

Figure 3. MRI before surgery. Intracranial thrombosis with failure to visualize the left sigmoid sinus and a flow reduction on the lateral sinus

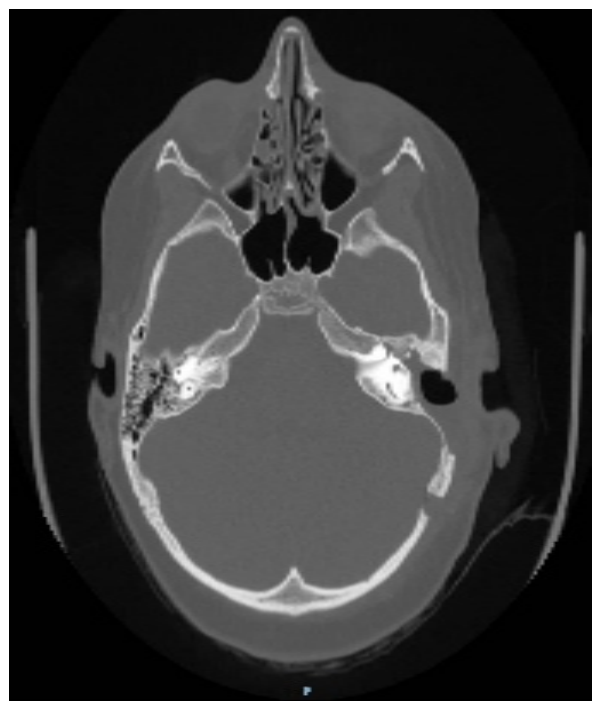

Figure 4. CT post surgery. The abscess was evacuated by a retrosigmoid approach, and a left mastoidectomy was performed

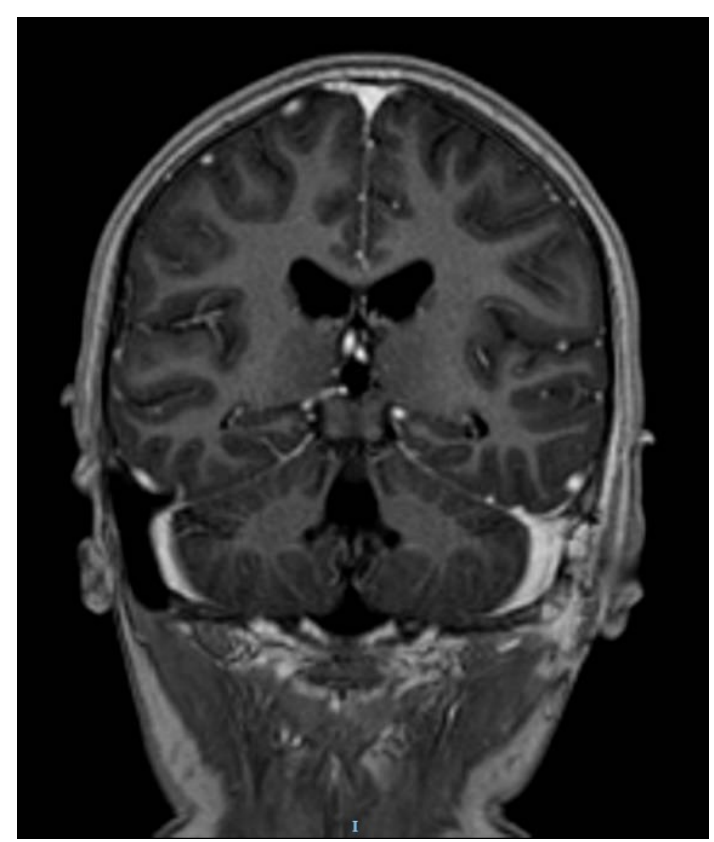

Figure 5. MRI 1 month post surgery. Recovery of cerebral vascular patency

usually supported by Pneumococcus and does not necessarily need to be treated surgically $[1,2]$. Our usual attitude is to hospitalize children with clinically suspicious otomastoiditis, where an Otorhinolaringologist find a post-auricular inflammation, with swelling, hyperemia or retroauricular pain [3]. Otoscopical evidence of otitis media with or without otorrhea is not sufficient to make the diagnosis of mastoiditis. Typically, these children are already taking oral antibiotic treatment with amoxicillin + clavulanic acid 3 times a day. Therefore, we administer high-dose intravenous antibiotics, usually starting with ceftriaxone.

The coalescent acute mastoiditis is the most common intratemporal complication of acute otitis media. Since the use of antibiotics, its frequency has decreased, but for unclear reasons the incidence seems to increase again in recent years [3].

From Literature we know that a quarter of pediatric patients with coalescent mastoiditis could present with a simultaneous intracranial complication [4]. In general the suspicion of a complication arises when hyperpyrexia, pain and general conditions of children do not improve in 24-48 hours, and above all when they become soporous. In this case we recommend to perform a CT scan, followed by a MRI if there is a suspicion of one intracranial complication [5]. When there is an intracranial complication, often the cause is an anaerobic organism. This element should not be forgotten when the most appropriate antibiotic therapy is to be decided. We agree with Zevallos et al. on the importance of collaborating with infectivologists for longterm intravenous antibiotic therapy in treating pediatric patients with advanced mastoiditis [4].

Another topic of discussion concerns the need to treat or not the acute otogenic lateral sinus thrombosis. This is a rare intracranial complication and its treatment is still controversial, because the recommended extent of surgical intervention and need for routine anticoagulation are unclear [6,7]. In fact the recanalization can occur spontaneously, and the clinical results seem to be independent from the achievement of a complete recanalization or not. Besides in Literature there are numerous episodes of intracranial haemorrhage, even fatal, in treated with anticoagulants patients [8]. 
In this case we reached a complete recovery of cerebral vascular patency at magnetic resonance control carried out 1 month after drainage and mastoidectomy (Figure 5). The young $\mathrm{Pz}$ has gone through a complete neurological healing.

\section{Conclusions}

Middle otitis is a very common infection, especially in the pediatric age. Large-spectrum antibiotic therapy does not always prevent coalescent mastoiditis. This more severe form of infection can be complicated by involvement of the intracranial structures, leading to cerebral abscess and lateral sinus thrombosis. Close collaboration between Otolaryngologist, Pediatrician and Infectivologist is essential in these cases, as much as long-term intravenous antibiotic therapy.

It is still questioned whether and especially when we have to resort to surgery. Another topic of discussion concerns the necessity or not of long-lasting anticoagulant therapy. In our opinion more extensive studies are needed to settle these doubts. However, it is essential that every doctor has clear the possible complications of a simple otitis media. No specialist can underrate an often mild, but never trivial, infection, such as otomasoiditis.

\section{References}

1. Paffetti A, D’Aviera L, Le Foche F, Mannozzi P, Mastropietro C, et al. (1998) Successful meropenem therapy of a brain abscess and meningitis arising from acute purulent otomastoiditis: case report. J Chemother 10: 132-135. [Crossref]

2. Atzeni M, Iozzi C, Pinna M, Benetti GL, Scazzeri F (2015) A case of complicated otomastoiditis. Pediatr Med Chir 37: pmc. [Crossref]

3. Palma S, Fiumana E, Borgonzoni M, Bovo R, Rosignoli M, et al. (2007) Acute mastoiditis in children: the "Ferrara" experience. Int J Pediatr Otorhinolaryngol 71: 1663-1669. [Crossref]

4. Zevallos JP, Vrabec JT, Williamson RA, Giannoni C, et al. (2009) Advanced pediatric mastoiditis with and without intracranial complications. Laryngoscope 119: 16101615. [Crossref]

5. Dobben GD, Raofi B, Mafee MF, Kamel A, Mercurio S (2000) Otogenic intracranial inflammations: role of magnetic resonance imaging. Top Magn Reson Imaging 11: 7686. [Crossref]

6. Funamura JL, Nguyen AT, Diaz RC (2014) Otogenic lateral sinus thrombosis: case series and controversies. Int J Pediatr Otorhinolaryngol. 78: 866-870. [Crossref]

7. Sitton MS, Chun R (2012) Pediatric otogenic lateral sinus thrombosis: role of anticoagulation and surgery. Int J Pediatr Otorhinolaryngol. 76: 428-432. [Crossref]

8. Rosenberg JD, Parikh SR (2011) Anticoagulation therapy as a supplement to recanalization for the treatment of sigmoid sinus thrombosis: a case report. Ear Nose Throat J 90: 418-422. [Crossref]

Copyright: (C2018 Amadei EM. This is an open-access article distributed under the terms of the Creative Commons Attribution License, which permits unrestricted use, distribution, and reproduction in any medium, provided the original author and source are credited. 